\title{
Neonatal Bone Marrow Transplantation in MPS IIIA Mice
}

\author{
Adeline A Lau $\cdot$ N. Jannah Shamsani • \\ Leanne K. Winner • Sofia Hassiotis • \\ Barbara M. King • John J. Hopwood • Kim M. Hemsley
}

Received: 13 April 2012 /Revised: 12 July 2012 / Accepted: 19 July 2012 / Published online: 10 August 2012

(C) SSIEM and Springer-Verlag Berlin Heidelberg 2013

\begin{abstract}
Patients with some neurological lysosomal storage disorders (LSD) exhibit improved clinical signs following bone marrow transplantation (BMT). The failure of mucopolysaccharidosis (MPS) type IIIA patients and adult mice with the condition to respond to this treatment may relate to factors such as impaired migration of donorderived cells into the brain, insufficient enzyme production and/or secretion by the donor-derived microglial cells, or the age at which treatment is initiated. To explore these possibilities, we treated neonatal MPS IIIA mice with whole unfractionated bone marrow and observed that nucleated blood cell reconstitution occurred to a similar
\end{abstract}

Communicated by: Ed Wraith

Competing interests: None declared

Electronic supplementary material: The online version of this article (doi:10.1007/8904_2012_169) contains supplementary material, which is available to authorized users.

A.A. Lau $(\varangle) \cdot$ N.J. Shamsani $\cdot$ L.K. Winner $\cdot$ S. Hassiotis B.M. King $\cdot$ J.J. Hopwood $\cdot$ K.M. Hemsley

Lysosomal Diseases Research Unit, SA Pathology at the Women's and Children's Hospital, 72 King William Rd,

North Adelaide 5006, Australia

e-mail: adeline.lau@adelaide.edu.au

N.J. Shamsani · J.J. Hopwood · K.M. Hemsley Department of Pediatrics, Adelaide University, Adelaide, Australia

A.A. Lau $\cdot$ N.J. Shamsani $\cdot$ J.J. Hopwood $\cdot$ K.M. Hemsley Center for Stem Cell Research, The Robinson Institute,

The University of Adelaide, Adelaide, Australia

Present Address:

N.J. Shamsani

Pediatrics Oncology Research Laboratory, Pediatrics Department, Medical Faculty, University Malaya Medical Centre, 50603,

Kuala Lumpur, Malaysia degree in MPS IIIA mice receiving green fluorescent protein (GFP)-expressing normal (treatment group) or MPS IIIA-GFP marrow (control group) and normal mice receiving normal-GFP marrow (control group). Further, similar distribution patterns of $\mathrm{GFP}^{+}$normal or MPS IIIA donor-derived cells were observed throughout the MPS IIIA mouse brain. We demonstrate that $N$-sulfoglucosamine sulfohydrolase (SGSH), the enzyme deficient in MPS IIIA, is produced and secreted in a manner proportional to that of other lysosomal enzymes. However, despite this, overall brain SGSH activity was unchanged in MPS IIIA mice treated with normal marrow and the lysosomal storage burden in whole brain homogenates did not decrease, most likely due to donor-derived cells comprising $<0.24 \%$ of total recipient brain cells in all groups. This suggests that the failure of MPS IIIA patients and mice to respond to BMT may occur as a result of insufficient donor-derived enzyme production and/or uptake by host brain cells.

\section{Introduction}

Lysosomal storage disorders (LSD) are a group of inherited conditions that most commonly result from the absence of a catabolic enzyme, causing the accumulation of partially degraded substrates. In Sanfilippo syndrome, or mucopolysaccharidosis (MPS) types IIIA, B, C or D, degradation of the glycosaminoglycan heparan sulfate is incomplete and as a result heparan sulfate-derived oligosaccharide fragments accumulate within the lysosome. Patients afflicted with Sanfilippo syndrome are generally diagnosed between 2-6 years of age and show a gradual deterioration of central nervous system function, increased aggression, hyperactivity, and sleep disturbance (Neufeld and Muenzer 2001). Several naturally occurring animal models of MPS IIIA have been 
identified, including a mouse model resulting from a missense mutation that causes an amino acid change from an aspartic acid to an asparagine at position 31 of the lysosomal enzyme $N$-sulfoglucosamine sulfohydrolase (SGSH; EC 3.10.1.1) (Bhaumik et al. 1999; Bhattacharyya et al. 2001). Congenic C57BL/6 MPS IIIA mice display reduced SGSH activity resulting in accumulation of heparan sulfate-derived disaccharides, GM2 and GM3 gangliosides and unesterified cholesterol, as well as behavioral changes such as reduced learning ability in the Morris Water Maze test, changes in open field activity, and altered anxietyrelated behaviors (Crawley et al. 2006; Fraldi et al. 2007; Lau et al. 2008, 2010).

Allogeneic hematopoietic stem/precursor cell transplantation (BMT) halts disease progression in several neurological LSD, such as MPS type I and metachromatic leukodystrophy, provided that a suitable donor match can be identified and that the transplant is undertaken early in the disease course (Shapiro et al. 1995). Despite the successful engraftment of donor cells, it appears that BMT in MPS IIIA patients is unable to improve or stabilize cognitive function (Klein et al. 1995; Sivakumur and Wraith 1999). Umbilical cord blood stem cell transplants have also been undertaken in Sanfilippo patients, with a 5 -year probability of survival of 56\% (Prasad et al. 2008). Two of the children treated at less than 2 years of age who survived the transplant may have had a modest improvement in cognitive skills, though were still developmentally delayed overall (Prasad et al. 2008). Pre-symptomatic MPS IIIA mice transplanted at 4 weeks of age show complete engraftment of donor cells in peripheral blood as well as high levels of SGSH activity and reduced heparan sulfate-derived oligosaccharide storage in the bone marrow compartment, measured using the disaccharide marker GlcNS-UA (Lau et al. 2010). While there was a reduction in brain GlcNS-UA of $27 \%$, this was insufficient to mediate a clinical improvement in the behavioral phenotype of the transplanted mice.

The basis of the differential response of patients with MPS IIIA and MPS I or metachromatic leukodystrophy to BMT is not known. One possibility is that the high concentration of undegraded heparan sulfate-derived fragments in host MPS IIIA tissues reduces the mobilization of donor bone marrow-derived cells into circulation, thereby resulting in fewer engrafted donor cells within the affected brain. Heparan sulfate proteoglycans modulate a wide variety of biological interactions, including the binding of several key molecules involved with hematopoietic stem cell homing and mobilization. For example, heparin, a highly sulfated form of heparan sulfate, forms tight complexes with neutrophil elastase and cathepsin $G$ which render these proteases $2-5$ times less active than their free counterparts toward natural and synthetic substrates (Frommherz et al. 1991). Considering that neutrophil Springer elastase and cathepsin $\mathrm{G}$ proteolysis mobilizes hematopoietic precursor/progenitor cells into circulation (Levesque et al. 2001), reduced activity of these enzymes due to interactions with heparan sulfate could potentially result in inefficient repopulation of donor-derived cells in blood and organs following BMT. Heparan sulfate-derived GlcNSUA is elevated 15 -fold in newborn MPS IIIA mouse brain, progressively increasing to maximal levels exceeding 250fold by 13 weeks of age (Crawley et al. 2006). Therefore, transplanting mice in the neonatal period, when GlcNS-UA is less abundant, may improve donor-derived cell mobilization and the efficacy of BMT. In the murine MPS VII model, BMT is more effective when delivered to neonatal compared to adult mice (Birkenmeier et al. 1991; Sands et al. 1993). Also, the clinical efficacy of cell-mediated therapies, such as BMT, is impacted by the ability of both the transplanted cells to produce and secrete enzyme containing mannose-6-phosphate moieties and the affected host cells to efficiently internalize this enzyme and target it toward the lysosome via the mannose-6-phosphate pathway (reviewed in Kornfeld 1992). In this study, we have examined several factors that may influence the outcome of BMT in MPS IIIA mice and the effect of neonatal transplantation on neuropathological storage.

\section{Materials and Methods}

\section{Mice and Genotyping}

All procedures were approved by the institutional Animal Ethics Committee and were conducted as per the guidelines of the National Health and Medical Research Council of

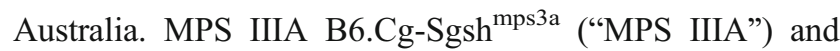
unaffected (wild-type or heterozygous; subsequently referred to as "normal") mice were obtained from a breeding colony maintained at the Women's and Children's Hospital (Crawley et al. 2006). The genotypes of the mice were determined from a toe clip collected at 5-7 days of age by extracting genomic DNA by overnight incubation at $37^{\circ} \mathrm{C}$ in $50 \mu \mathrm{L}$ of $50 \mathrm{mM}$ Tris, $\mathrm{pH} 8.0,2 \mathrm{mM} \mathrm{NaCl}, 1 \mathrm{mM}$ EDTA, 0.5\% (v/v) Tween20, and 0.5\% (v/v) Triton X-100 supplemented with $1.2 \mathrm{mg} / \mathrm{mL}$ proteinase $\mathrm{K}$. After heating at $95{ }^{\circ} \mathrm{C}$ for $10 \mathrm{~min}, 1-2 \mu \mathrm{L}$ of clarified lysate was amplified with forward primer $5^{\prime}$-NNT CTG TCT TCC TCA GCG-3', reverse primer 5'-GAT AAG GCT GTG GCG GGA CAG GG-3' (final concentration of $4 \mathrm{ng} / \mu \mathrm{L}$ of each primer), $0.2 \mathrm{mM}$ dNTP (Roche, Mannheim, Germany), and 1.25 U HotStarTaq DNA Polymerase (Qiagen, Doncaster, Australia) in a $50 \mu \mathrm{L}$ reaction by denaturing at $94^{\circ} \mathrm{C}$ for $15 \mathrm{~min}$ followed by 35 cycles of $94^{\circ} \mathrm{C}$ for $45 \mathrm{~s}, 55^{\circ} \mathrm{C}$ for $45 \mathrm{~s}$, and $72^{\circ} \mathrm{C}$ for $40 \mathrm{~s}$, and a 5 min final extension at $72^{\circ} \mathrm{C}$. After visualization of the $105 \mathrm{bp}$ PCR product, amplified DNA was digested with $5 \mathrm{U}$ 
Aci (New England Biolabs, MA, USA) at $37{ }^{\circ} \mathrm{C}$ before electrophoresis through a $4.5 \%(\mathrm{w} / \mathrm{v})$ agarose gel. As the G91A mutation abolishes an $A c i$ I site, wild-type mice display bands at 74,16 , and 15 bp while MPS IIIA mice display bands at 90 and $15 \mathrm{bp}$. Heterozygote mice display bands at $90,74,16$, and $15 \mathrm{bp}$.

Breeding pairs of C57BL/6-Tg(UBC-GFP)30Scha/J mice (Schaefer et al. 2001) were purchased from the Jackson Laboratory (ME, USA) and a pedigreed breeding colony was maintained at the Women's and Children's Hospital. These mice express green fluorescent protein (GFP) under the control of the human ubiquitin $\mathrm{C}$ promoter and wild-type levels of SGSH and are subsequently referred to as "normal-GFP" mice. Mice were confirmed as GFP-positive by viewing a toe clip under ultraviolet light.

A normal-GFP dam $\left(\mathrm{SGSH}^{+/+} \mathrm{GFP}^{+/+}\right)$was mated with an MPS IIIA stud $\left(\mathrm{SGSH}^{-1-} \mathrm{GFP}^{-/-}\right)$to generate offspring heterozygous for the $S G S H$ and GFP genes. The F1 animals were inter-crossed and the resultant F2 offspring were assessed for GFP expression (see "Flow Cytometry" section) and normal, carrier, or affected $S G S H$ gene status. A brother/ sister founder pair of MPS IIIA mice homozygous for the GFP allele ("MPS IIIA-GFP"; $\mathrm{SGSH}^{-/-} \mathrm{GFP}^{+/+}$) were used to establish a pedigreed colony.

\section{Neutrophil Elastase and Cathepsin G Activity}

Following $\mathrm{CO}_{2}$-mediated euthanasia, bone marrow extracellular fluid was extracted from 6-week-old normal and MPS IIIA mice by flushing the hind-leg bones with $0.5 \mathrm{~mL}$ ice-cold phosphate-buffered saline (PBS) using a $21 \mathrm{G}$ needle ( $n=9-10$ mice per group). For neutrophil elastase activity, $10 \mu \mathrm{L}$ of sample was diluted with $90 \mu \mathrm{L}$ of $0.1 \mathrm{M}$ Tris- $\mathrm{HCl}, \mathrm{pH} 7.5,0.5 \mathrm{M}$ sodium chloride, and $0.01 \%$ (v/v) sodium azide and then mixed with an equal volume of $800 \mu \mathrm{M}$ MeOSuc-Ala-Ala-Pro-Val-pNA substrate (Calbiochem, Australia). For cathepsin $\mathrm{G}$ activity, $10 \mu \mathrm{L}$ of sample was diluted in $90 \mu \mathrm{L}$ of $0.1 \mathrm{M}$ Tris- $\mathrm{HCl}, \mathrm{pH} 8.3,0.01 \%$ (v/v) sodium azide and mixed with $100 \mu \mathrm{L}$ of $800 \mu \mathrm{M}$ Suc-Ala-Ala-Pro-Phe-pNA (Calbiochem, Australia). Standard curves were prepared using recombinant neutrophil elastase (Calbiochem, Australia; 0-128 mU/mL) or cathepsin G (Calbiochem, Australia; 0-20 mU/mL). Following a 3-h incubation at $37{ }^{\circ} \mathrm{C}$, the absorbance was measured on a Victor $3^{\mathrm{TM}}$ multilabel reader (Perkin Elmer) at $405 \mathrm{~nm}$ to quantitate the amount of cleaved pNA. Protease activity was normalized to total protein content as determined by bicinchoninic acid assay (Pierce, IL).

\section{Transplantation and Necropsy}

Donor normal-GFP or MPS IIIA-GFP mice (8-10 weeks old) were treated with 5 -fluorouracil $(150 \mathrm{mg} / \mathrm{kg}$ intraperitoneal; Sigma, MO, USA). Three days later, the mice were euthanized and donor bone marrow cells were harvested by flushing the hind-leg bones with Dulbecco's modified Eagle's medium (DMEM) containing 2\% fetal calf serum (FCS) and $50 \mathrm{U}$ penicillin $/ 0.05 \mathrm{mg} / \mathrm{mL}$ streptomycin (Sigma, MO, USA) using a $23 \mathrm{G}$ needle (Lau et al. 2010). The cells were passed through a $100 \mu \mathrm{m}$ cell strainer and red blood cells were lysed with Gey solution (155 $\mathrm{mM}$ ammonium chloride, $10 \mathrm{mM}$ potassium hydrogen carbonate) at $37^{\circ} \mathrm{C}$ for $10 \mathrm{~min}$. Viable cells were counted with trypan blue dye (Sigma, MO, USA) and resuspended in PBS containing 30 U/mL heparin. Normal and MPS IIIA recipients (3 days old) were placed into a polypropylene container subdivided into $2.5 \mathrm{~cm} \times 2.5 \mathrm{~cm}$ compartments and irradiated with $4 \mathrm{~Gy}$ using a megavoltage linear accelerator at a dose rate of $2 \mathrm{~Gy} / \mathrm{min}$. The pups then received $1-2 \times 10^{6}$ donor cells (in $30 \mu \mathrm{L}$ total volume) intravenously via the superficial temporal vein. In the pilot study, 3-day-old mice were irradiated with 6 Gy prior to transplantation.

At 8 weeks post-transplant, the recipient mice were overdosed with $\mathrm{CO}_{2}$ and whole blood collected via cardiac puncture and transferred to an EDTA-coated vacuette. The brain was removed, divided along the midline and one hemisphere was further sectioned in the coronal plane into five 2-mm thick slices and frozen at $-20{ }^{\circ} \mathrm{C}$. The other hemisphere was post-fixed in $4 \%$ paraformaldehyde in PBS at $4{ }^{\circ} \mathrm{C}$ for $24 \mathrm{~h}$, cryo-protected in $30 \%(\mathrm{w} / \mathrm{v})$ sucrose in PBS (Sigma, MO, USA) and embedded in Tissue-Tek OCT compound (Tokyo, Japan).

\section{Flow Cytometry}

The $-/-,-/+$, or $+/+$ status of the GFP gene during the generation of the MPS IIIA-GFP strain was determined in up to $20 \mu \mathrm{L}$ of blood collected from the saphenous vein using $4 \%(\mathrm{w} / \mathrm{v})$ EDTA-treated capillary tubes. The percentage of donor cell reconstitution in leukocytes was determined in duplicate samples of $50 \mu \mathrm{L}$ whole blood taken at euthanasia (Lau et al. 2010). Erythrocytes were lysed in $2 \mathrm{~mL}$ of FACS lysing solution (BD Biosciences, NJ, USA). The leukocytes were blocked with IntraGam ${ }^{\circledR} \mathrm{P}$ (CSL Ltd, Parkville, Australia), labeled with PE-Cy5-conjugated anti-CD45 (1:10 dilution; BD Biosciences, NJ, USA) and then washed with $0.5 \%(\mathrm{w} / \mathrm{v})$ bovine serum albumin (Sigma, MO, USA) in IsoFlow Sheath Flow (Beckman Coulter, CA, USA). The cells were then analyzed on a FACSCalibur flow cytometer (Beckton Dickson, NJ, USA) equipped with CellQuest software (version 3.1).

SGSH Activity and GlcNS-UA Measurement in Tissue Homogenates

Livers, spleens, and brain tissues (slice 2) were homogenized in $500 \mu \mathrm{L}$ of $20 \mathrm{mM}$ Tris, $500 \mathrm{mM}$ sodium chloride, $\mathrm{pH} 7.4$, and sonicated twice for $30 \mathrm{~s}$ each. Samples for SGSH activity 
measurement were dialyzed overnight in $200 \mathrm{mM}$ sodium acetate, $\mathrm{pH}$ 5.2, and incubated with $400 \mathrm{pmol}$ of a tritiated, heparin-derived tetrasaccharide substrate (Hopwood and Elliott 1982) at $60^{\circ} \mathrm{C}$. The amount of substrate and product were separated and quantified by high-performance liquid chromatography and normalized to total protein content (MicroBCA kit; Pierce, IL, USA).

The relative amount of a disaccharide marker (GlcNSUA) of heparan sulfate storage was determined in brain samples from experimental mice or from untreated MPS IIIA brain as an internal control $(50 \mu \mathrm{g}$ total homogenate per sample). The tissues were derivatized with 1-phenyl-3methyl-5-pyrazolone (Sigma, MO, USA) and assessed by liquid chromatography electrospray ionization tandem mass spectrometry analysis using a PE Sciex API 4000 QTRAP triple quadrupole mass spectrometer with a turbo spray source, as previously described (Hemsley et al. 2009). The intra-assay coefficient of variation of the quality control brain homogenate was $4.9 \%$.

\section{Quantitative Real-Time PCR}

Genomic DNA was extracted from brain slices 3 and 5 according to published methods (Joshi et al. 2008), except that DNA was precipitated with $0.1 \mathrm{x}$ volume of $3 \mathrm{M}$ sodium acetate and $2 \times$ volumes of $100 \%$ ethanol. The purity and concentration of DNA was determined at $260 \mathrm{~nm}$ using a Nanodrop (ND-1000, version 3.7.0; Thermo Scientific, Scoresby, Australia).

Primer Express Software (version 3; Applied Biosystems, CA, USA) was used to design EGFP forward (5'-GACGACGGCAACTACAAGAC-3') and reverse (5'-GTCCTCCTTGAAGTCGATGC-3') primers and hypoxanthine guanine phosphoribosyl transferase (HPRT) forward (5'-GTGGGAATGCGCAATCACT-3') and reverse (5'-TCCACTCTTCAGGTGGAAAATAGG-3') primers. The efficiency $(E)$ of each primer set was determined using 10 -fold dilutions of normal-GFP genomic DNA (0.05 to $500 \mathrm{ng}$ ) and was calculated from the slope of the standard curve (cycle threshold $(\mathrm{Ct})$ against log genomic DNA concentration) using the formula $E=-1+10^{(-1 / \text { slope })}$.

Real-time quantitative PCR reactions were carried out with an Applied Biosystems 7300 Real-Time PCR System in 96-well clear optical reaction plates with optical adhesive covers (Applied Biosystems, CA, USA). Reactions (30 $\mu \mathrm{L})$ were performed in triplicate, with $100 \mathrm{ng}$ genomic DNA, SYBR $^{\circledR}$ Green PCR master mix (Applied Biosystems, CA, USA) and either EGFP $(3 \mu \mathrm{M})$ or HPRT primers $(10 \mu \mathrm{M})$. The cycling conditions employed were $50{ }^{\circ} \mathrm{C}$ for $2 \mathrm{~min}$, $95{ }^{\circ} \mathrm{C}$ for $10 \mathrm{~min}$, followed by 40 cycles of $95{ }^{\circ} \mathrm{C}$ for $15 \mathrm{~s}$ and $60{ }^{\circ} \mathrm{C}$ for $1 \mathrm{~min}$. $\mathrm{Ct}$ values were determined using the 7000 Sequence Detection software (v1.3; Applied Biosystems, CA, USA). The relative number of EGFP copies versus HPRT copies was determined using the method of Pfaffl (2001) and expressed as a percentage of GFP-expressing cells compared to a reference untreated normal-GFP mouse brain (100\% GFP-positive).

GM3 Ganglioside Immunohistochemistry and GFP Visualization

Tissues were processed, imaged, and assessed in a single batch by an experimenter without knowledge of the genotype/treatment status of the samples. Frozen brain sections $(6 \mu \mathrm{m}$ thick) were cut in the sagittal plane and collected onto Superfrost Plus slides (Menzel-Glaser, Braunschweig, Germany). GM3 ganglioside secondary storage was quantitated by staining with a monoclonal anti-GM3 antibody (Seikagaku Biobusiness Corporation, Tokyo, Japan; 1:750) using published methods (Lau et al. 2010). For GFP imaging, cryo-sections were rinsed in PBS and mounted with Vectashield mounting media containing 4',6-diamidino-2-phenylindole (DAPI) to label all nuclei (Vector Laboratories, CA, USA). Images were captured using an Olympus Colorview Soft Imaging System and an Olympus BX41 (GM3 ganglioside) or BX61 (GFP) microscope, and the percentage of immunostained area was determined with AnalySIS Lifescience software (version 2.8, Build1235, Olympus Soft Imaging Solutions).

\section{Cell Culture}

All cells were derived from neonatal mice euthanized by decapitation at 0-4 days of age. Skin fibroblast cultures (Villegas and McPhaul 2005) were grown in DMEM/ Nutrient Mixture F-12 Ham (Sigma, MO, USA) with 20\% FCS (ThermoElectron Corporation, Melbourne, Australia), $2 \mathrm{mM}$ glutamine (SAFC Biosciences, KS, USA) and $50 \mathrm{U}$ penicillin $/ 0.05 \mathrm{mg} / \mathrm{mL}$ streptomycin (Sigma, MO, USA). Mixed neural cells derived from whole brain (Sutherland et al. 2008) were maintained in DMEM (Sigma, MO, USA) with 5\% FCS (ThermoElectron Corporation, Melbourne, Australia), 5\% horse serum (Institute of Medical and Veterinary Science, Adelaide, Australia), $2 \mathrm{mM}$ glutamine (SAFC Biosciences, KS, USA), and $50 \mathrm{U}$ penicillin/ $0.05 \mathrm{mg} / \mathrm{mL}$ streptomycin (Sigma, MO, USA). Cells were cultured at $37{ }^{\circ} \mathrm{C}$ with $5 \% \mathrm{CO}_{2}$.

\section{Lysosomal Enzyme Activity Assays}

Neural cells and fibroblasts were cultured until confluence. The cells were then given a media change and cultured for 1 week without further media changes. Conditioned media was collected and stored frozen and control unconditioned media samples were also included. The cells were detached with $10 \%$ trypsin (SAFC Biosciences, KS, USA) in PBS 
(Sigma, MO, USA), centrifuged at $6,240 \mathrm{~g}$ for $5 \mathrm{~min}$ and resuspended in $300 \mu \mathrm{L}$ of $20 \mathrm{mM}$ Tris, $500 \mathrm{mM}$ sodium chloride, and $\mathrm{pH}$ 7.4. Samples were subjected to six cycles of freezing/thawing in a slurry of dry ice and ethanol.

SGSH activity was determined by mixing $8 \mu \mathrm{L}$ of sample

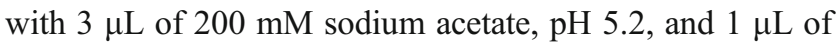
tritiated, heparin-derived tetrasaccharide substrate (400 pmol) and incubating for $16 \mathrm{~h}$ at $60{ }^{\circ} \mathrm{C}$. The conversion of substrate into product was then measured as described for brain homogenates. For all other enzyme assays, $10 \mu \mathrm{L}$ of sample was mixed with $40 \mu \mathrm{L}$ of $0.9 \% \mathrm{NaCl}$ and $50 \mu \mathrm{L}$ of the appropriate substrate and incubated at $37{ }^{\circ} \mathrm{C}$. After 1 $\mathrm{h}(\alpha$-mannosidase and $\alpha$-fucosidase), $2 \mathrm{~h}$ ( $\alpha$-iduronidase; IDUA), or $4 \mathrm{~h}$ ( $\alpha$ - $N$-acetylglucosaminidase; NAGLU), the reaction was quenched with $1.5 \mathrm{~mL}$ glycine buffer (200 mM glycine, $125 \mathrm{mM}$ sodium carbonate, $\mathrm{pH}$ 10.7) and the amount of free 4-methylumbelliferone (4MU) was measured on a Perkin Elmer LS-50B spectrofluorometer. The substrates used were 4MU-2-acetomido-2-deoxy- $\alpha$-Dglucopyranoside (NAGLU; $2 \mathrm{mM}$ in $200 \mathrm{mM}$ sodium acetate, $\mathrm{pH}$ 4.3; Toronto Research Chemicals, North York, Canada), 4MU- $\alpha$-L-idopyranosiduronic acid (IDUA; $2 \mathrm{mM}$ in formate buffer, $\mathrm{pH}$ 3.6; Toronto Research Chemicals, North York, Canada), 4MU- $\alpha$-mannopyranoside ( $\alpha$-mannosidase; $4 \mathrm{mM}$ in acetate buffer, $\mathrm{pH}$ 4.0; Melford Laboratories, Chelsworth, UK), and 4MU- $\alpha$-L-fucopyranoside ( $\alpha$-fucosidase; $1 \mathrm{M}$ in citrate phosphate buffer, $\mathrm{pH}$ 5.0; Melford Laboratories, Chelsworth, UK).

The activity in unconditioned media controls was subtracted from activity measured in the conditioned media samples to determine the secreted activity in the media. The calculated activity in each sample was then corrected for total protein content to give a total amount of intracellular or extracellular activity. Secreted enzyme activity was calculated as the percentage of extracellular activity as a proportion of the sum of intracellular and extracellular activity.

\section{GlcNS-UA Determination in Co-cultured Normal and MPS IIIA Cells}

Normal-GFP and MPS-GFP fibroblasts (2 weeks postconfluent) were plated into 6-well trays at various ratios of normal and MPS IIIA cells, with each well containing $5 \times 10^{6}$ cells in total, based on a previous report that the number of microglia in the brain is thought to remain constant in adulthood (Lawson et al. 1992) and therefore in a transplantation setting the donor microglia would replace recipient microglia rather than supplement microglial numbers. To prevent clonal dominance of normal-GFP cells in the co-cultured populations, the cells were detached after $48 \mathrm{~h}$ with $10 \%$ trypsin (SAFC Biosciences, KS, USA) in PBS (Sigma, MO, USA), centrifuged at $6,240 \mathrm{~g}$ for $5 \mathrm{~min}$ and resuspended in $150 \mu \mathrm{L}$ of $20 \mathrm{mM}$ Tris, $500 \mathrm{mM}$ sodium chloride, $\mathrm{pH}$ 7.4. The cells were sonicated twice for $30 \mathrm{~s}$ and the total protein content was quantitated using a MicroBCA kit (Pierce, IL, USA). The relative amount of GlcNS-UA was determined in $100 \mu \mathrm{g}$ of cell extract, as described for brain homogenates. The intra-assay coefficient of variation of an internal quality control sample of cultured cells was $2.6 \%$.

\section{Statistics}

Data are expressed as the mean \pm SEM. Data were analyzed using one-way ANOVA with GraphPad Prism software (v.502) with post hoc comparisons made using the Bonferroni correction factor or with unpaired t-tests. Statistical significance was considered to be $P<0.05$.

\section{Results}

Donor Leukocyte Engraftment Following Myeloreduction

Three-day-old normal and MPS IIIA pups were transplanted with syngeneic GFP-expressing mononuclear bone marrow cells following irradiation with $4 \mathrm{~Gy}$. The three treatment groups were (1) normal recipients receiving normal-GFP donor cells ( $n=9$ mice; 8 male, 1 female), (2) MPS IIIA recipients receiving MPS IIIA donor cells ( $n=5$ mice; 4 male, 1 female), and (3) MPS IIIA recipients receiving normal-GFP donor cells ( $n=7$ mice; 3 male, 4 female). The body weights of the three cohorts were indistinguishable at 2 months post-transplantation $(P>0.05$; Fig. 1a). Quantitation of donor-derived peripheral blood leukocyte reconstitution revealed no significant differences in mobilization between the transplanted groups of mice, with an average of $25 \%, 24 \%$ and $21 \%$ $\mathrm{CD} 45^{+} \mathrm{GFP}^{+}$donor cells measured at 8 weeks posttransplantation in control normal mice, control MPS IIIA mice, and treated MPS IIIA mice, respectively (Fig. 1b). Further, the catalytic activity of two serine proteases involved in the mobilization of hematopoietic precursor/ progenitor cells was determined in bone marrow extracellular fluid from 6-week-old mice. Although there was a trend toward an increase in neutrophil elastase activity in MPS IIIA mice, statistically significant differences were not measured $(P>0.05$; Fig. 1c). Likewise, no significant differences in cathepsin $\mathrm{G}$ activity were measured between genotype groups $(P>0.05$; Fig. 1c). 

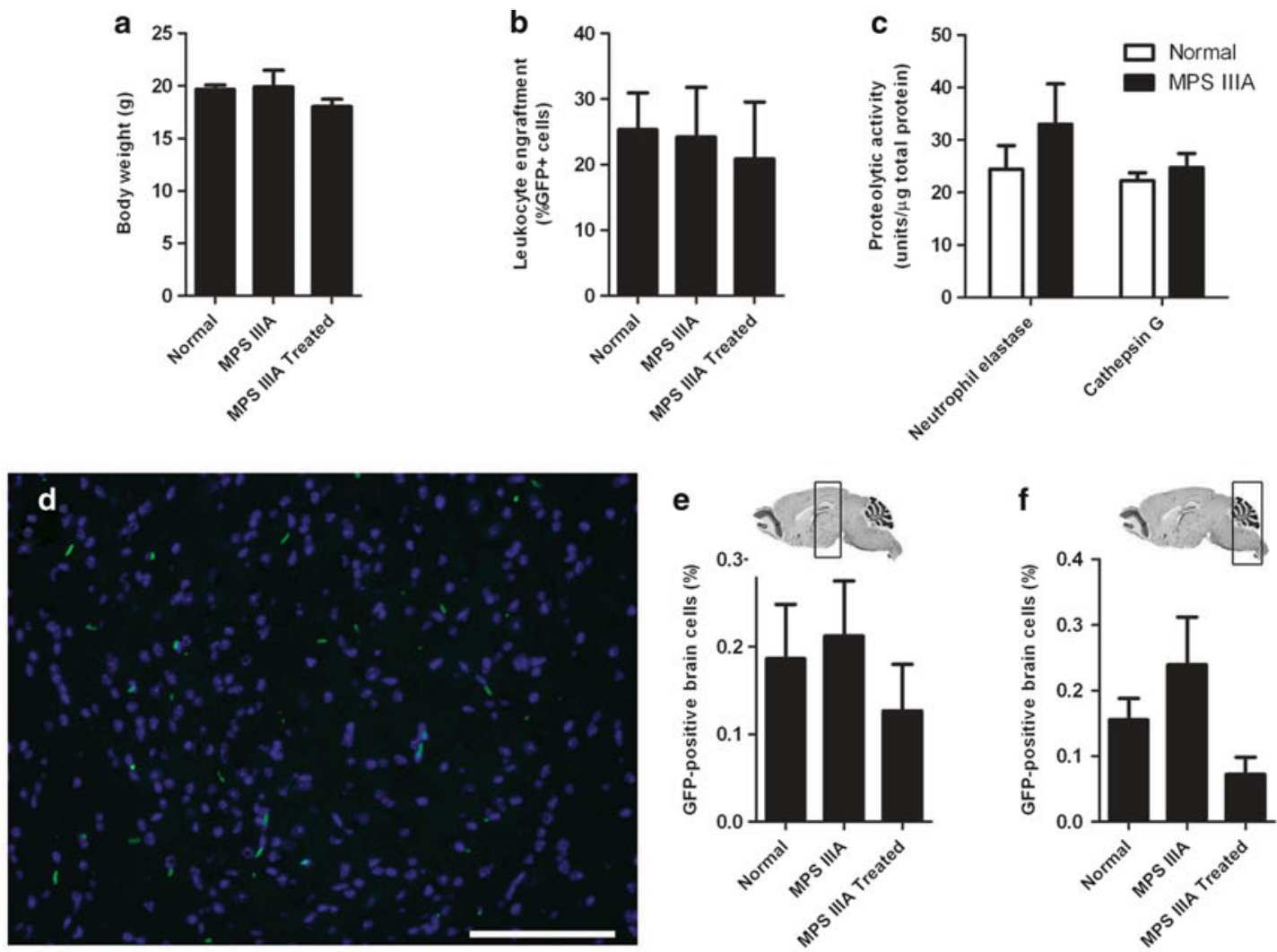

g

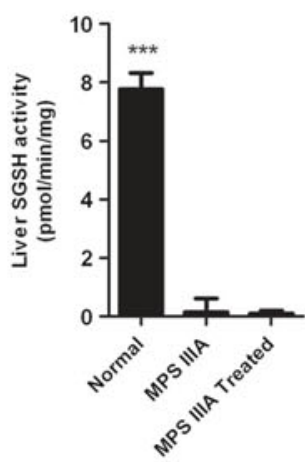

j

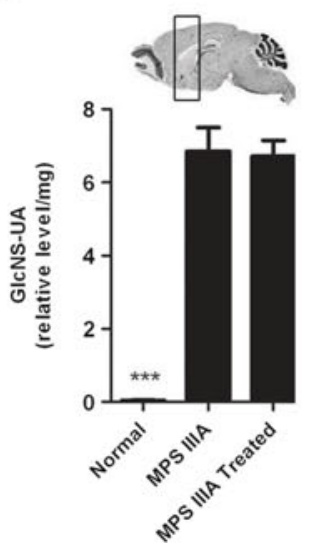

Fig. 1 Effect of transplantation of neonatal MPS IIIA mice preconditioned with 4 Gy. (a) The weight of MPS IIIA recipient mice h

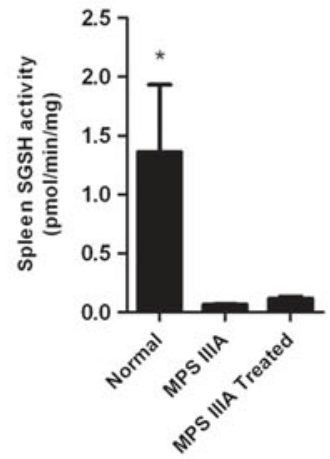

k

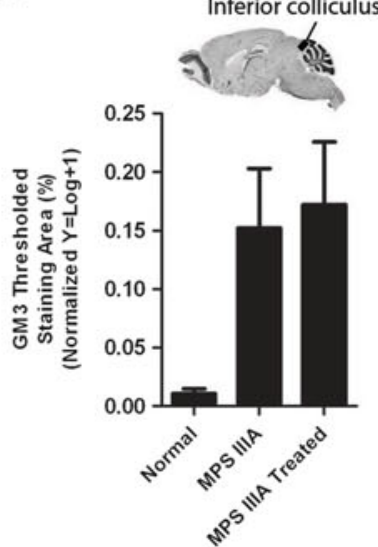

i
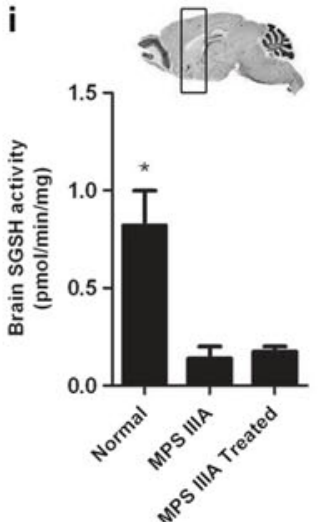

I

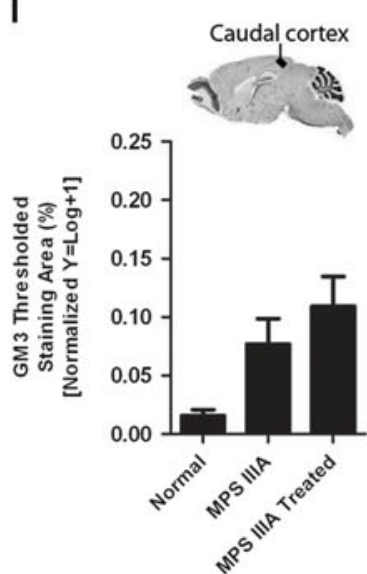

\section{黑 Springer}

transplanted with normal-GFP donor bone marrow ("MPS IIIA Treated"; $n=7$ mice) and transplanted control groups of normal 
GFP-Expressing Donor Cells Repopulate Throughout the Brain, but at Low Levels

To verify that donor-derived cells had successfully migrated and engrafted within the central nervous system, GFPexpressing cells were visualized in brain tissue sections. GFP-positive cells were widely distributed throughout the brains of all groups of transplanted mice, including regions such as the cerebellum, cerebral cortex, hippocampus, thalamus, inferior colliculus, superior colliculus, and brainstem (Fig. 1d and Supplementary Figure 1). To obtain a quantitative measure of the number of donor-derived cells in the brain, the relative GFP transgene copy number (compared to the HPRT gene as an endogenous control) was determined in hemi-coronal brain slices by quantitative real-time PCR and compared to untreated transgenic mouse brains that expressed GFP in all nucleated cells (i.e., all cells except for red blood cells). Initial validation experiments showed that the amplification efficiency was similar for the GFP and HPRT primer sets (101.6\% and 95.4\%, respectively). In addition, dissociation curve analysis demonstrated the formation of a single peak for each primer set, with a melting temperature of $84^{\circ} \mathrm{C}$ and $80{ }^{\circ} \mathrm{C}$ for GFP and HPRT primers, respectively.

The relative number of GFP transgene copies was examined in brain slices 3 and 5 of transplanted mice by real-time quantitative PCR. In brain slice 3 , the relative level of GFP repopulation was, on average, $0.1-0.2 \%$, with no difference detected between treatment/genotype groups (Fig. 1e). The maximum percentage of GFP engraftment measured in the brain was $0.52 \%$ in a normal transplanted control mouse. A similar low level of donor cell repopulation was measured in brain slice $5(0.1-0.2 \%$ on average; Fig. 1f).

Effect of Transplantation on SGSH Activity and Substrate Accumulation

To determine the effect of donor cell transplantation, SGSH activity was measured in liver, spleen, and brain homogenates. In the liver, MPS IIIA mice transplanted with MPS IIIA bone marrow displayed $1.8 \%$ of the SGSH activity measured in normal mice transplanted with normal bone marrow (Fig. 1g). Transplantation of normal-GFP bone marrow cells in MPS IIIA mice had no impact on liver SGSH activity (1.3\% of wild-type SGSH activity). Similar trends were observed in the spleen (Fig. 1h) and brain parenchyma (Fig. 1i), with transplantation of normal-GFP cells into MPS IIIA recipients yielding no significant differences in SGSH activity compared to control-transplanted MPS IIIA mice.

Heparan sulfate-derived GlcNS-UA was considerably elevated in the brain of control transplanted MPS IIIA mice receiving MPS IIIA-GFP bone marrow relative to normal mice transplanted with normal-GFP donor cells (165-fold normal; $P<0.0001$; Fig. 1j). Transplantation of normalGFP bone marrow did not significantly reduce the relative level of GlcNS-UA in MPS IIIA recipient mouse brain (162-fold normal).

The effect of treatment on secondary storage products was also examined at 8 weeks post-transplant. In both the inferior colliculus (Fig. 1k) and caudal cortex (Fig. 11), numerous GM3 ganglioside-immunoreactive puncta were observed in MPS IIIA mice transplanted with MPS IIIA-GFP bone marrow. In contrast, GM3 ganglioside immunoreactivity was infrequently observed in the transplanted normal control mice. Delivery of normal-GFP donor bone marrow to MPS IIIA recipient mice had no observable treatment effect on the amount of GM3 ganglioside immunoreactivity (Fig. 11-k).

The Percentage of Extracellular SGSH Activity is Similar to Other Lysosomal Enzymes

We next examined whether the proportion of secreted SGSH and NAGLU (EC 3.2.1.50; deficient in MPS IIIB) in wild-type cells was altered compared to the hydrolases deficient in MPS I, $\alpha$-mannosidosis, and $\alpha$-fucosidosis (i.e., neurodegenerative LSD that are successfully treated by allogeneic BMT). In skin fibroblast cultures, SGSH activity
Fig. 1 (continued) mice receiving normal-GFP cells ("Normal"; $n=9$ mice) and MPS IIIA mice receiving MPS IIIA cells ('MPS IIIA'; $n=5$ mice) was recorded at 8 weeks post-treatment. (b) The percentage of donor-derived $\mathrm{GFP}^{+} \mathrm{CD}^{+} 5^{+}$leukocytes was measured by flow cytometry. (c) The catalytic activity of two proteolytic enzymes that are involved with the mobilization of bone marrow cells into circulation was measured using chromogenic substrates in bone marrow extracellular fluid harvested from untreated normal (open bars) and MPS IIIA (filled bars) mice ( $\mathrm{n}=9$-10 mice per group). (d) Cryo-sections were examined for the presence of donor-derived GFP-expressing cells (green) compared to the total number of DAPI-stained cell nuclei (blue) in the cerebellum. Scale bar is $100 \mu \mathrm{m}$. The relative GFP transgene copy number (compare to the HPRT gene as an endogenous control) was determined via quantitative real-time PCR in transplanted mouse (e) brain slice 3 and (f) slice 5. An untreated transgenic normal-GFP mouse brain was used as a calibrating sample $(100 \%)$. (g) Liver, (h) spleen, and (i) brain SGSH activity was measured in tissue homogenates using a radiolabeled tetrasaccharide substrate and HPLC separation. (j) The relative amount of GlcNS-UA disaccharide was determined by tandem mass spectrometry as a measure of the amount of primary storage material in the transplanted mouse brain. The effect of treatment on GM3 ganglioside storage in the brain was quantitated in the $(\mathbf{k})$ inferior colliculus and (I) caudal cortex using immunohistological methods and AnalySIS Lifescience software. All data are expressed as the mean \pm SEM. $* P<0.05, * * * P<0.001$ versus the control-transplanted MPS IIIA control group 
was reduced in cell extracts compared to all other assayed lysosomal enzymes (Fig. 2a). Similar trends were observed in neural cells and in media samples of both cell types, although not all differences reached statistical significance (Fig. 2b-d). The proportion of extracellular SGSH activity was comparable to the percentage of extracellular NAGLU and $\alpha$-iduronidase (EC 3.2.1.76) activity in both cell populations and was significantly higher than that of $\alpha$-fucosidase (EC 3.2.1.51; Fig. 2e-f). $\alpha$-Mannosidase (EC 3.2.1.24) was the only lysosomal enzyme that was measured at a significantly higher percentage of extracellular activity compared to SGSH (Fig. 2e-f).

Preconditioning with Higher Irradiation Doses Improves Donor Cell Engraftment but Induces Acute Radiation Syndrome Symptoms

In an attempt to increase the degree of donor-derived cell engraftment in affected organs, including the brain, 3-day-old normal mouse pups were preconditioned with a higher myeloablative dose of 6 Gy before normal-GFP donor cell infusion ( $n=4$ mice) and compared to a second cohort of normal mice irradiated with 4 Gy and transplanted with normal-GFP cells $(n=5$ mice). At 4 weeks post-transplant, analysis of $\mathrm{CD} 5^{+} \mathrm{GFP}^{+}$peripheral blood leukocytes showed that the donor-type chimerism was $10 \pm 3 \%$ in mice preconditioned with 4 Gy. Donor cell engraftment was significantly improved following preconditioning with $6 \mathrm{~Gy}$, with $37 \pm 4 \% \mathrm{CD}^{4} 5^{+} \mathrm{GFP}^{+}$donor cells measured $(P<0.001)$. As observed in the earlier study, mice irradiated with 4 Gy showed no signs of acute radiation syndrome. In contrast, recipient pups that received $6 \mathrm{~Gy}$ displayed significant growth retardation at 4 weeks posttreatment $(P<0.05)$, weighing $28 \%$ less than mice irradiated with $4 \mathrm{~Gy}$, and also developed ataxia and involuntary tremors. Due to the severity of the side effects of the preconditioning regime in the latter group of mice, the study was terminated and the mice euthanized at 4 weeks of age.

\section{Reduction of Substrate in MPS IIIA Cultured Cells After Co-incubation with Normal Cells}

Previously, we achieved a $27 \%$ reduction in brain GlcNSUA when approximately $3 \%$ of the total brain cells were of donor origin (unpublished data and Lau et al. 2010). Cells of macrophage/microglial origin have been estimated to make up to $12 \%$ of total brain cells (Lawson et al. 1990), which raised the following question: if a BMT regime was able to replace all host microglia with donor cells, what level of amelioration of stored GlcNS-UA is achievable in the transplanted MPS IIIA mouse brain? We cultured MPS IIIA cells $(0 \%$ normal cells $)$ and determined the relative level of GlcNS-UA accumulating within them. Additional cultures were established so that they contained $90 \%$ MPS IIIA cells and $10 \%$ normal cells or $80 \%$ MPS IIIA cells and $20 \%$ normal cells, engraftment levels that may be feasible if all microglia were donor-derived. We anticipated that SGSH released from the normal cells would be internalized by MPS IIIA cells using mannose-6-phosphate receptorbased mechanisms, subsequently mediating a reduction in GlcNS-UA in the MPS IIIA cells.

We observed that co-culture of MPS IIIA cells with $10 \%$ normal cells mediated a statistically significant reduction of $14.5 \%$ in GlcNS-UA (Fig. 3). This consisted of a $5 \%$ reduction from cross-correction of the MPS IIIA cells by the input normal cells with the remainder due to a reduced amount of input GlcNS-UA by the addition of fewer MPS IIIA cells. Also, co-culture with $20 \%$ normal cells enabled a $37 \%$ total reduction within the timeframe of the experiment (21\% derived from cross-correction of MPS IIIA cells). These data are consistent with our in vivo findings (Lau et al. 2010) and suggest that even if all MPS IIIA brain microglia/macrophages were able to be replaced with unaffected donor-derived cells, the level of stored heparan sulfate-derived substrates may not be reduced beyond approximately one-third.

\section{Discussion}

We evaluated the therapeutic efficacy of BMT in neonatal (3day-old) MPS IIIA mice. This equates to approximately midway through the second trimester of a human fetus in terms of brain development (Clancy et al. 2001). A myeloreductive preconditioning regime $\left(4 \mathrm{~Gy}, 1 \times 10^{6}\right.$ donor cells delivered intravenously) enabled replacement of approximately $25 \%$ of $\mathrm{CD} 45^{+}$leukocytes of donor origin and did not result in measurable increases in SGSH activity in visceral organs or the brain. Subsequently, there was no effect of treatment on primary or secondary storage compounds in the brain. Likewise, transplantation of MPS IIIB mice at 2-4 days of age after irradiation with 2 Gy did not result in a significant increase in brain NAGLU activity or ameliorate neuropathology (Heldermon et al. 2010). These data contrast with those from MPS VII mice, where transplantation resulted in $\sim 4 \%$ of wild-type $\beta$-glucuronidase activity, focal reductions in lysosomal vacuolization in the brain and normalization of auditory-evoked brainstem responses (Sands et al. 1993, 1995). Given that this is a knockout model, all of the measured $\beta$-glucuronidase would be newly synthesized enzyme. Even greater reductions in lysosomal storage were observed when higher irradiation doses were utilized (Sands et al. 1993). Improvements in the accelerating rotor-rod test were also evident in Twitcher mice, a model of globoid-cell leukodystrophy, transplanted at 3 days of age ( $4 \mathrm{~Gy} ; 1 \times 10^{6}$ donor cells), 

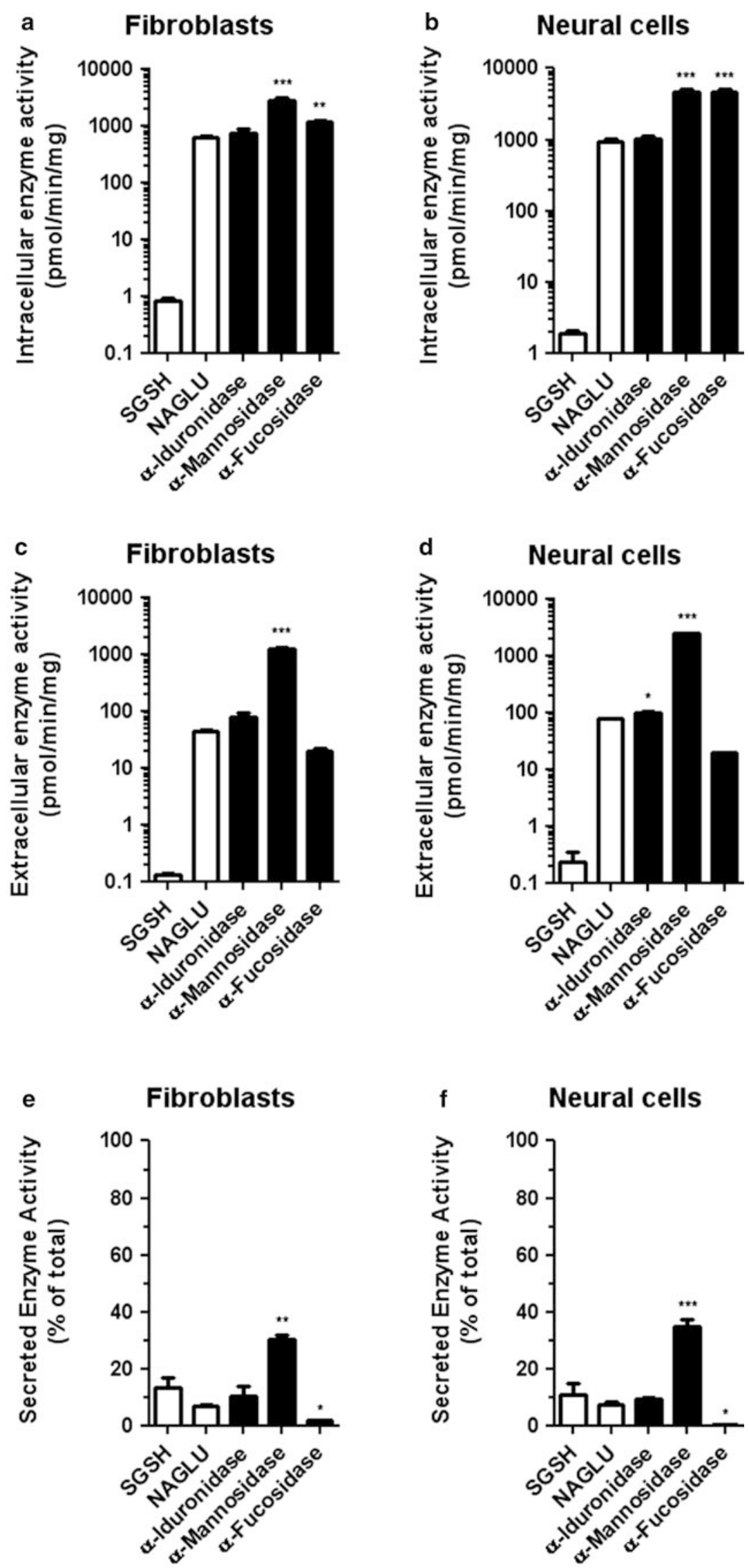

Fig. 2 (continued) 


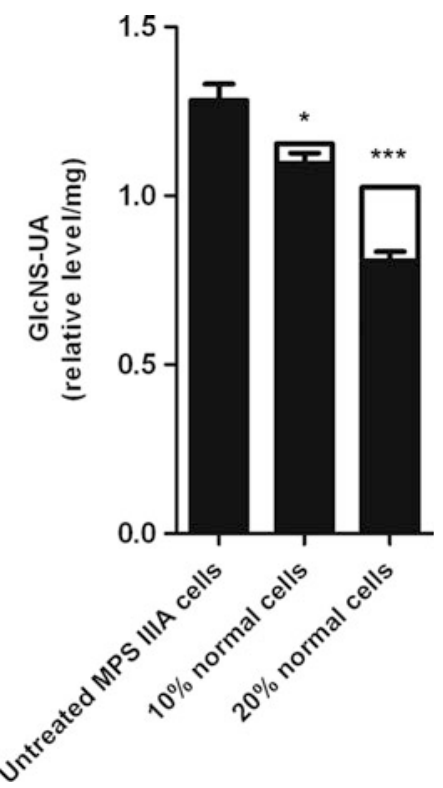

Fig. 3 GlcNS-UA storage in co-cultured normal and MPS IIIA fibroblasts. Normal-GFP and MPS-GFP cells $\left(5 \times 10^{6}\right.$ cells in total $)$ were co-cultured for $48 \mathrm{~h}$ at different ratios. The relative amount of GlcNS-UA was then determined by tandem mass spectrometry and compared to the untreated group (100\% MPS IIIA; filled bars). The calculated maximum amount of GlcNS-UA in the MPS IIIA cell portion of each treatment group due to dilution with the normal-GFP cells is denoted by the open bars (i.e., $90 \%$ or $80 \%$ of the relative levels of measured GlcNS-UA measured in the 100\% MPS IIIA cultures). Data are expressed as the mean \pm SEM. ${ }^{*} P<0.05$, $* * * P<0.001$ versus the untreated group

despite brain galactosylceramidase activity not being significantly increased following treatment (Lin et al. 2007). Twitcher mice transplanted at 8-11 days of age (9 Gy; $3-5 \times 10^{7}$ donor cells delivered intraperitoneally) generated up to $15 \%$ normal levels of galactosylceramidase activity in the brain, which was sufficient to prevent hindlimb paralysis (Hoogerbrugge et al. 1988). It is evident that MPS IIIA disease-related factors impair or reduce the capacity for clinical efficacy to be achieved with BMT in human MPS III types A, B, and C (Klein et al. 1995; Shapiro et al. 1995) and murine MPS III types A and B (Zheng et al. 2004; Heldermon et al. 2010; Lau et al. 2010), and the growing body of experimental data strongly suggest that BMT with unmodified cells should not be considered as a treatment for MPS III.

So why does BMT stabilize neurocognitive function in some LSD but is unable to resolve the disease pathology in other disorders? In our study, the SGSH activity generated under these transplantation conditions was insufficient to correct primary and secondary storage, which could potentially be explained by a number of reasons. First, inadequate migration of donor-derived cells into the brain following transplantation would reduce the number of healthy cells able to produce SGSH within the brain. It is well-established that bone marrow-derived cells are capable of migrating across the blood-brain barrier after myeloablative conditioning and then trans-differentiate into microglia, the resident macrophage population of the brain (e.g., Cogle et al. 2004; Simard and Rivest 2004). Microglia represent between 5\% and $12 \%$ of total brain cells, dependent on the brain region (Lawson et al. 1990), and considering that proliferation of resident microglia also contributes up to half of the brain macrophage pool (Lawson et al. 1992), the absolute maximum proportion of donor cells that can migrate and repopulate the brain is less than $12 \%$. Between $0.1-0.2 \%$ of recipient brain cells were found to be of donor origin in the brain of neonatally-transplanted MPS IIIA mice, and our attempt to raise the percentage of donor leukocytes in peripheral blood from $25 \%$ (and subsequently increase the proportion of donor cells within the brain) by increasing the irradiation preconditioning dose to 6 Gy resulted in acute radiation syndrome. Increased chimerism may be achieved using increased cell doses or alternate irradiation protocols (e.g., divided dose of 6 Gy). Also, the assessment of additional groups at longer times post-transplant could possibly have increased the number of donor-derived cells within the brain.

Quantitative real-time PCR analysis of brain tissue from adult MPS IIIA mice transplanted in a previous study (where 93\% donor-type leukocyte chimerism was achieved) revealed that donor cells represented only $2.8 \pm 1.2 \%$ of brain cells (unpublished data and Lau et al. 2010). BMT did not improve the behavioral phenotype of these treated MPS IIIA mice. The low level of donor cell repopulation in the MPS IIIA mouse brain is unlikely to be the result of a defect in mobilization of bone marrow-derived cells from the bone marrow compartment to peripheral blood, as the activity of two serine proteases involved in cellular mobilization was similar in normal and MPS IIIA mice. These proteases, cathepsin $\mathrm{G}$ and neutrophil elastase, have been implicated in altered bone marrow progenitor cell retention/mobilization in sialidosis mice (Yogalingam et al. 2008). Additionally, no differences in GFP-expressing donor leukocyte reconstitution
Fig. 2 (continued) Lysosomal enzyme activity in wild-type cells. (a, c, e) Murine skin fibroblasts or $(\mathbf{b}, \mathbf{d}, \mathbf{f})$ mixed neural cell cultures were harvested at 7 days post-confluence. The amount of $(\mathbf{a}, \mathbf{b})$ intracellular enzyme activity in cell extracts and (c, d) extracellular enzyme activity in conditioned media samples was determined using fluorogenic or radiolabeled substrates for SGSH, NAGLU, $\alpha$ iduronidase, $\alpha$-mannosidase, and $\alpha$-fucosidase. LSD that do not respond to BMT are depicted by open bars while LSD where BMT stabilizes neurocognitive decline are depicted by filled bars. $(\mathbf{e}, \mathbf{f})$ The percentage of extracellular activity as a percentage of the total measured activity for each enzyme is shown. All data are expressed as the mean \pm SEM. ${ }^{*} P<0.05,{ }^{*} P<0.01,{ }^{* * *} P<0.001$ versus SGSH activity 
or the percentage of $\mathrm{GFP}^{+}$brain cells were measured between normal and MPS IIIA recipients, suggesting that movement of donor cells from the vasculature to the brain was unimpaired in the disease state. This contrasts with the increased brain repopulation in transplanted metachromatic leukodystrophy mice (Biffi et al. 2004) and may be one of the factors contributing to the successful correction of neurological symptoms in this disorder.

It is possible that even if all microglia were normal in their enzyme content, this would still be insufficient to generate enough enzyme activity to mediate clinical improvements. This concept is supported by data generated in mouse models of Gaucher disease that either expressed glucosylceramidase activity in the microglia (and skin) or in skin fibroblasts only (Enquist et al. 2007). Both models showed neurological abnormalities, with only marginal delays in the onset of symptoms and minimal impact on life span. Further, we demonstrated that even co-culture of MPS IIIA cells with $20 \%$ normal cells (a level that cannot be achieved by transplantation alone) is unable to normalize GlcNS-UA storage. Co-incubation of approximately equal numbers of MPS I and MPS II cells (where the MPS I cells provide the corrective enzyme for the MPS II cells and vice versa) for 48 hours normalizes sulfated glycosaminoglycan storage (Neufeld and Cantz 1971). Further studies are warranted to elucidate whether this level of storage reduction can be achieved in MPS IIIA cells after co-incubation with higher doses of normal cells and the degree of primary storage reduction that is required for an impact on clinical symptoms.

Second, correction of MPS IIIA cells relies on the effective production and secretion of mannose-6-phosphorylated SGSH by the donor cells and subsequent cation-independent mannose-6-phosphate receptor (CI-MPR)-mediated uptake of SGSH by the neighboring host cells, and thus inadequate SGSH production, secretion, or endocytosis may also contribute to the lack of therapeutic benefit in transplanted MPS IIIA mice. SGSH exhibited the lowest activity in the intracellular and extracellular fractions of wild-type cells and this may relate to differences in assay sensitivity (natural substrate for SGSH versus 4MU-conjugated substrates) or the natural abundance of the enzymes. However, as each ratio was measured under identical assay conditions, our calculation of the ratio of intracellular versus secreted enzyme remains valid. No relationship was evident between the percentage of secreted lysosomal enzyme and the efficacy of BMT, with $\alpha$-mannosidase and $\alpha$-fucosidase (enzymes deficient in LSD that respond to BMT) displaying the highest and lowest percentage of extracellular enzyme activity, respectively. This suggests that pathological changes in the relative proportion of secreted SGSH are not the primary cause for the lack of correction in MPS IIIA. It remains to be ascertained whether the CI-MPR is mis-localized in host MPS IIIA cells, thus reducing the efficiency of exogenous SGSH uptake by affected cells. Pompe disease fibroblasts showed less efficient uptake of recombinant $\alpha$-glucosidase enzyme due to reduced CI-MPR bioavailability at the plasma membrane, with the extent of abnormalities closely correlating with disease severity (Cardone et al. 2008). Increasing the amount of SGSH produced via ex vivo modification of bone marrowderived cells with a gene therapy vector may overcome this issue (Zheng et al. 2004; Langford-Smith et al. 2012).

In conclusion, BMT was ineffective at ameliorating primary and secondary storage in the MPS IIIA mouse brain despite transplantation occurring in the neonatal period. Further studies are required to elucidate the mechanism(s) that result in differential responses to transplantation amongst LSD to direct the design of an effective BMT-mediated treatment for MPS IIIA.

Acknowledgments We thank the staff of the Department of Radiation Oncology at the Royal Adelaide Hospital for irradiating the mice; Ms Hanan Hannouche for genotyping and performing some of the flow cytometry during the generation of the MPS IIIA-GFP mice; Ms Amanda Luck for mouse provision and assistance with irradiation; Dr Litsa Karageorgos, Mr Kristian Brion, and Dr Anthony Fedele for advice on quantitative PCR assay development; Dr Maria Fuller for assistance with the mass spectrometry; Dr Michael Fietz from the National Referral Laboratory for Lysosomal, Peroxisomal and Related Genetic Disorders, SA Pathology, for advice regarding the lysosomal enzyme activity assays and access to the spectrofluorometer; and the Department of Haematology, SA Pathology, for access to the flow cytometer. This work was supported by the Fondation Sanfilippo Suisse to AAL, KMH, and JJH. The authors confirm that the content of the chapter has not been influenced by the sponsors.

\section{Synopsis}

Despite intervention in the neonatal period and demonstration of unimpaired mobilization, engraftment, and secretion of lysosomal $\mathrm{N}$-sulfoglucosamine sulfohydrolase enzyme by normal cells, bone marrow transplantation does not improve neuropathology in the murine MPS IIIA brain.

\section{References}

Bhattacharyya R, Gliddon B, Beccari T et al (2001) A novel missense mutation in the lysosomal sulfamidase is the basis of MPS III A in a spontaneous mouse mutant. Glycobiology 11:99-103

Bhaumik M, Muller VJ, Rozaklis T et al (1999) A mouse model for mucopolysaccharidosis type III A (Sanfilippo syndrome). Glycobiology 9:1389-1396

Biffi A, De Palma M, Quattrini A et al (2004) Correction of metachromatic leukodystrophy in the mouse model by transplantation of genetically modified hematopoietic stem cells. J Clin Invest 113:1118-1129

Birkenmeier EH, Barker JE, Vogler CA et al (1991) Increased life span and correction of metabolic defects in murine mucopoly- 
saccharidosis type VII after syngeneic bone marrow transplantation. Blood 78:3081-3092

Cardone M, Porto C, Tarallo A et al (2008) Abnormal mannose-6phosphate receptor trafficking impairs recombinant alpha-glucosidase uptake in Pompe disease fibroblasts. Pathogenetics 1:6

Clancy B, Darlington RB, Finlay BL (2001) Translating developmental time across mammalian species. Neuroscience 105:7-17

Cogle CR, Yachnis AT, Laywell ED et al (2004) Bone marrow transdifferentiation in brain after transplantation: a retrospective study. Lancet 363:1432-1437

Crawley AC, Gliddon BL, Auclair D et al (2006) Characterization of a C57BL/6 congenic mouse strain of mucopolysaccharidosis type IIIA. Brain Res 1104:1-17

Enquist IB, Bianco CL, Ooka A et al (2007) Murine models of acute neuronopathic Gaucher disease. Proc Natl Acad Sci U S A 104:17483-17488

Fraldi A, Hemsley K, Crawley A et al (2007) Functional correction of CNS lesions in a MPS-IIIA mouse model by intracerebral AAVmediated delivery of sulfamidase and SUMF1 genes. Hum Mol Genet 16:2693-2702

Frommherz KJ, Faller B, Bieth JG (1991) Heparin strongly decreases the rate of inhibition of neutrophil elastase by alpha 1-proteinase inhibitor. J Biol Chem 266:15356-15362

Heldermon CD, Ohlemiller KK, Herzog ED et al (2010) Therapeutic Efficacy of Bone Marrow Transplant, Intracranial AAV-mediated Gene Therapy, or Both in the Mouse Model of MPS IIIB. Mol Ther 18:873-880

Hemsley KM, Luck AJ, Crawley AC et al (2009) Examination of intravenous and intra-CSF protein delivery for treatment of neurological disease. Eur J Neurosci 29:1197-1214

Hoogerbrugge PM, Poorthuis BJ, Romme AE et al (1988) Effect of bone marrow transplantation on enzyme levels and clinical course in the neurologically affected twitcher mouse. J Clin Invest 81:1790-1794

Hopwood JJ, Elliott H (1982) Diagnosis of Sanfilippo A syndrome by estimation of sulphamidase activity using a radiolabelled tetrasaccharide substrate. Clin Chim Acta 123:241-250

Joshi M, Keith Pittman H, Haisch C et al (2008) Real-time PCR to determine transgene copy number and to quantitate the biolocalization of adoptively transferred cells from EGFP-transgenic mice. Biotechniques 45:247-258

Klein K, Krivit W, Whitley C et al (1995) Poor cognitive outcome of eleven children with Sanfilippo syndrome after bone marrow transplantation and successful engraftment. Bone Marrow Transplantation 15:S176-S181

Kornfeld S (1992) Structure and function of the mannose 6-phosphate/ insulinlike growth factor II receptors. Annu Rev Biochem 61:307-330

Langford-Smith A, Wilkinson FL, Langford-Smith KJ et al (2012) hematopoietic stem cell and gene therapy corrects primary neuropathology and behavior in mucopolysaccharidosis IIIA Mice. Mol Ther doi:10.1038/mt.2012.82

Lau AA, Crawley AC, Hopwood JJ et al (2008) Open field locomotor activity and anxiety-related behaviors in mucopolysaccharidosis type IIIA mice. Behav Brain Res 91:130-136

Lau AA, Hannouche H, Rozaklis T et al (2010) Allogeneic stem cell transplantation does not improve neurological deficits in mucopolysaccharidosis type IIIA mice. Exp Neurol 225:445-454
Lawson LJ, Perry VH, Dri P et al (1990) Heterogeneity in the distribution and morphology of microglia in the normal adult mouse brain. Neuroscience 39:151-170

Lawson LJ, Perry VH, Gordon S (1992) Turnover of resident microglia in the normal adult mouse brain. Neuroscience 48:405-415

Levesque JP, Takamatsu Y, Nilsson SK et al (2001) Vascular cell adhesion molecule-1 (CD106) is cleaved by neutrophil proteases in the bone marrow following hematopoietic progenitor cell mobilization by granulocyte colony-stimulating factor. Blood 98:1289-1297

Lin D, Donsante A, Macauley S et al (2007) Central nervous systemdirected AAV2/5-mediated gene therapy synergizes with bone marrow transplantation in the murine model of globoid-cell leukodystrophy. Mol Ther 15:44-52

Neufeld EF, Cantz MJ (1971) Corrective factors for inborn errors of mucopolysaccharide metabolism. Ann N Y Acad Sci 179:580-587

Neufeld EF, Muenzer J (2001) The mucopolysaccharidoses. In: Scriver CR (ed) The metabolic and molecular bases of inherited diseases. 8th ed. New York: McGraw-Hill, pp 3421-3452

Pfaffl MW (2001) A new mathematical model for relative quantification in real-time RT-PCR. Nucleic Acids Res 29:e45

Prasad V, Mendizabal A, Parikh S et al (2008) Unrelated donor umbilical cord blood transplantation for inherited metabolic disorders in 159 pediatric patients from a single center: influence of cellular composition of the graft on transplantation outcomes. Blood 112:2979-2989

Sands MS, Barker JE, Vogler C et al (1993) Treatment of murine mucopolysaccharidosis type VII by syngeneic bone marrow transplantation in neonates. Lab Invest 68:676-686

Sands MS, Erway LC, Vogler C et al (1995) Syngeneic bone marrow transplantation reduces the hearing loss associated with murine mucopolysaccharidosis type VII. Blood 86:2033-2040

Schaefer BC, Schaefer ML, Kappler JW et al (2001) Observation of antigen-dependent CD8+ T-cell/ dendritic cell interactions in vivo. Cell Immunol 214:110-122

Shapiro EG, Lockman LA, Balthazor M et al (1995) Neuropsychological outcomes of several storage diseases with and without bone marrow transplantation. J Inherit Metab Dis 18:413-429

Simard AR, Rivest S (2004) Bone marrow stem cells have the ability to populate the entire central nervous system into fully differentiated parenchymal microglia. Faseb J 18:998-1000

Sivakumur P, Wraith JE (1999) Bone marrow transplantation in mucopolysaccharidosis type IIIA: a comparison of an early treated patient with his untreated sibling. J Inherit Metab Dis 22:849-850

Sutherland LS, Hemsley KM, Hopwood JJ (2008) Primary culture of neural cells isolated from the cerebellum of newborn and adult mucopolysaccharidosis type IIIA mice. Cell Mol Neurobiol 28:949-959

Villegas J, McPhaul M (2005) Establishment and culture of human skin fibroblasts. Curr Protoc Mol Biol Chapter 28:Unit 283.

Yogalingam G, Bonten EJ, van de Vlekkert D et al (2008) Neuraminidase 1 is a negative regulator of lysosomal exocytosis. Dev Cell 15:74-86

Zheng Y, Ryazantsev S, Ohmi K et al (2004) Retrovirally transduced bone marrow has a therapeutic effect on brain in the mouse model of mucopolysaccharidosis IIIB. Mol Genet Metab 82:286-295 